\title{
L199, A NEW VARIABLE STAR IN M13
}

\author{
VIOLAT-BORDONAU, FRANCISCO \\ Observatorio Astronómico Norba Caesarina, 10195, Cáceres, Spain, fviolat@gmail.com
}

\begin{abstract}
During a routine study of the variable stars of Messier 13 (NGC $6205=\mathrm{Cl}$ $1639+365$ ) we have discovered the variability of L199 (TYC 2588-1386-2), a red giant star member of the cluster: using the photometric data -in $V$ band- obtained in the 2019 and 2020 campaigns we have verified its variability. Data from the Zwicky Transient Facility (ZTF) and Deras et al. (2019) were utilized to determine its type of variability and period estimation: our data suggest that it is a semiregular red giant, similar to the other variables of this type of the cluster, whose cycles of photometric variation are not identical; we derived a period of $\sim 27$ days (although without a regular periodicity) and an amplitude smaller: $0.08 \pm$ 0.03 magnitudes in $V$ band although they may be somewhat larger or smaller. With this discovery the cluster now has 63 variable stars.
\end{abstract}

\section{Introduction}

Ludendorff 199 (Ludendorff, 1905), hereafter L199 (TYC 2588-1386-2 and 2MASS $\mathrm{J} 16413253+3624424$ ) is a red giant star located in Messier 13 from Gaia's parallax (BailerJones et al., 2021), one of the brightest ( $V \sim 5.8$ mag.) and best known of the globular clusters in the constellation of Hercules ( $\alpha=16 \mathrm{~h} 41^{\prime} 41.24$ "', $\delta=+36^{\circ} 27^{\prime} 35.5^{\prime \prime}, \mathrm{J} 2000$ ); the distance to the cluster was estimated as $7.1 \pm 0.1 \mathrm{kpc}$, with an average metallicity of $[\mathrm{Fe} / \mathrm{H}]=-1.58 \pm$ 0.09 and 12.6 Gyrs (Deras et al., 2019). Photographically L199 it is a moderately bright amber-colored star similar to other stars in the cluster: it is located on one of the "spider legs", a set of arc-shaped alignments formed by the brightest stars in the cluster (magnitude 12.1-12.5 V). In the literature it appears with magnitude 12.21 in $V$ band (Osborn, 2000), 12.175 $\mathrm{V}$ in the paper of Sandquist et al. (2010) and 12.177 $\mathrm{V}$ in the paper of Stetson et al. (2019). In none of the numerous photometric studies carried out on the cluster is it mentioned that it is variable: in the paper by Welty (1985) no variability was detected up to its detection limit ( $>0.2$ magnitudes in $B$ band); in the paper by Lupton et al. (1987) three radial velocities measurements are tabulated from which it was concluded that it is not variable; in Osborn's paper (2000) it does not appear as a variable either, being its standard deviations $\sigma$ equal to 0.02 in band $V$ and 0.03 in $B$ : if it was a variable star its amplitudes $\Delta V$ and $\Delta B$ would be very small ( $<0.1$ magnitudes). In the paper by Heinze et al. (2018) it appears as dubious although they provide a period of the variation as $\mathrm{P}=24.86$ days; Deras et al. (2019) they do not include it among the new variable stars they have discovered in the cluster. Finally, Stetson et al. (2019) indicate that its variability index (the probability that it is a variable star) is equal to 5.484 with a weight equal to 107.5 : with these two values it 
should be a variable star. Our photometric measurements, as well as two other similar data sets (ZTF photometry and Deras et al., 2019), confirm this.

\section{Observation an data reduction}

Since the spring of 2019 we have studied the variable stars of Messier 13: we employed the 0.2-m telescope of the Observatorio Astronómico Norba Caesarina, at Cáceres, Spain, located at $455 \mathrm{~m}$ above sea level, to obtain time-series imaging of the globular cluster. The image data were obtained during several runs between 2019 June and 2020 October, where we collected a total of 507 (2019) and 741 images (2020) through Johnson $V$ filter, respectively (see Table 1 for a detailed log of the observations): the exposure times were always 120 s. The CCD camera is a Starlight Xpress MXV-7, of $752 \times 580$ pixels, with a scale of $0.90 \% /$ pixel and a field of view of $11.4 \times 8.5 \mathrm{arcmin}^{2}$ (Figure 1). The calibration of the frames consisted of the bias and dark substraction as well as the flat-field correction.

Table 1: Observational log

\begin{tabular}{ccccc} 
Year & H.J.D. Start & H.J.D. End & Nights & Images \\
\hline 2019 & 2458670.581 & 2458767.330 & 98 & 507 \\
2020 & 2459003.670 & 2459136.336 & 134 & 741
\end{tabular}

The photometric data were reduced using the software FOTODIF ${ }^{1}$ (FOTOmetría DIFerencial, differential photometry) and calibrated using three stars of very well determined $B$ and $V$ magnitudes (Table 2) published in the photometric study by Sandquist et al. (2010). The CCD finder chart (with labelled stars) is shown in Figure 1: the magnitudes were determined relative to L199, L745 and L158, whose constancy during the run was confirmed using L77 (mag. 12.762 V), L353 (mag. 12.812 V) and L1073 (mag. 12.856 V). Table 2 presents the coordinates (2000.0) of the comparison and check stars taken from SIMBAD and their $V$ magnitudes and $B-V$ colour index from Sanquist et al. (2010). This calibration procedure with three stars works perfectly: in Figure 2 we present the light curves of the variable stars V11 (upper panel) and V17 (lower panel) throughout the 2020 campaign; in both we can see the behavior of both stars over the weeks, and both are totally coherent without showing any strange or unexpected effect on them.

1 Written by Julio Castellano, http://www.astrosurf.com/orodeno/fotodif/index.htm 


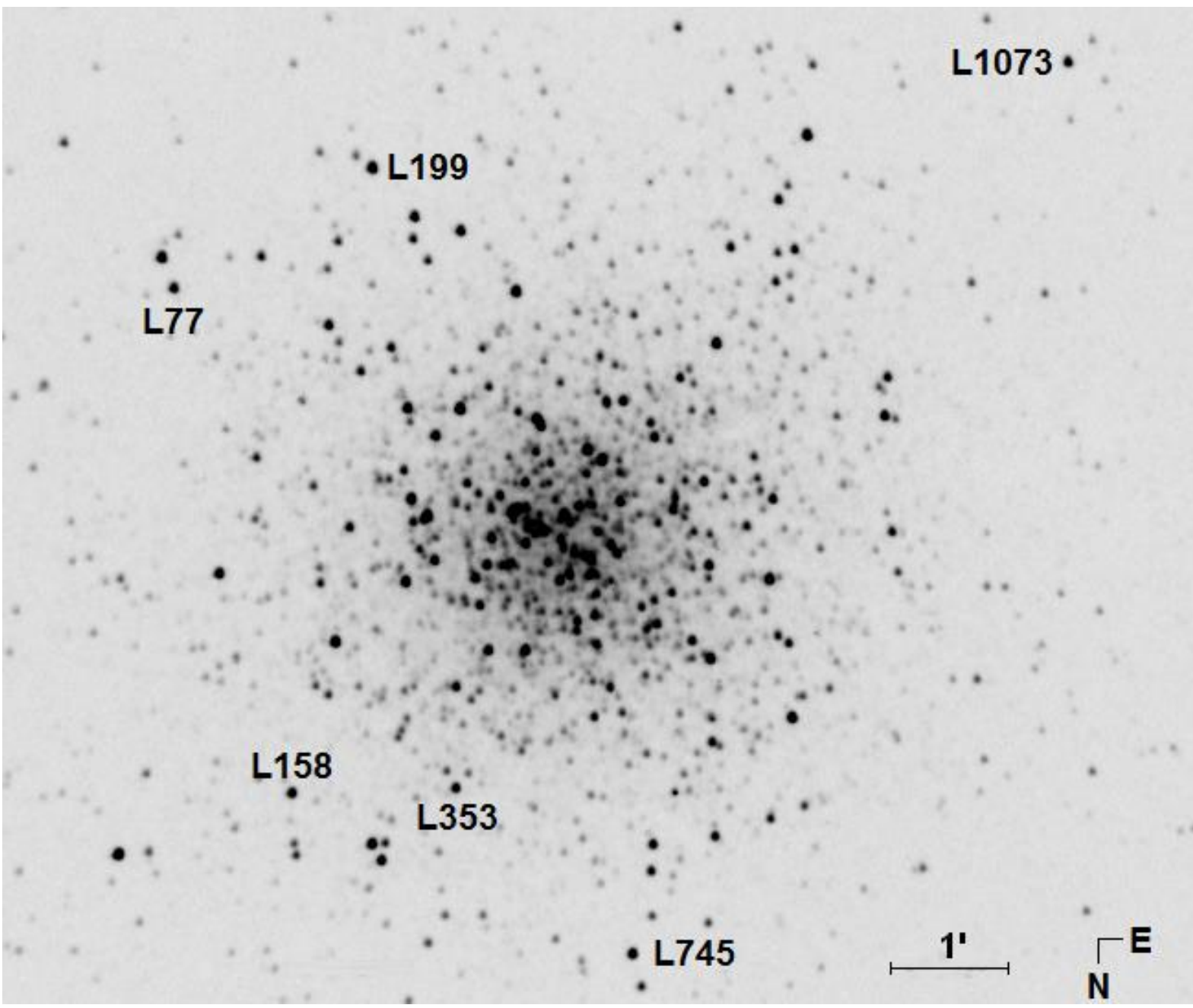

Figure 1: The CCD observed field of Messier 13 with labeled stars: L199 (the new variable), L745, L158, L77, L353 and L1073. North is down, East to the right.

Table 2: Comparison and check stars

\begin{tabular}{lccccc} 
Star & ID & $\begin{array}{c}\text { RA (J2000) } \\
{[\mathrm{h}: \mathrm{m}: \mathrm{s}]}\end{array}$ & $\begin{array}{c}\text { DEC (J2000) } \\
{\left[{ }^{\circ}{ }^{\prime} \text { "] }\right.}\end{array}$ & $\begin{array}{c}V \\
{[\mathrm{mag}]}\end{array}$ & $\begin{array}{c}B-V \\
{[\mathrm{mag}]}\end{array}$ \\
\hline \hline L199 & TYC 2588-1386-2 & $16: 41: 32.53$ & $+36: 24: 42.57$ & 12.175 & 1.363 \\
L745 & 2MASS J16414486+3630514 & $16: 41: 44.85$ & $+36: 30: 51.37$ & 12.480 & 1.293 \\
L158 & 2MASS J16413053+3629434 & $16: 41: 30.52$ & $+36: 29: 43.44$ & 12.686 & 1.144 \\
L77 & 2MASS J16412464+3625449 & $16: 41: 24.63$ & $+36: 25: 45.11$ & 12.762 & 1.141 \\
L353 & 2MASS J16413725+3629368 & $16: 41: 37.24$ & $+36: 29: 36.77$ & 12.812 & 1.138 \\
L1073 & 2MASS J16420085+3623338 & $16: 42: 00.84$ & $+36: 23: 33.67$ & 12.856 & 1.087 \\
\hline
\end{tabular}



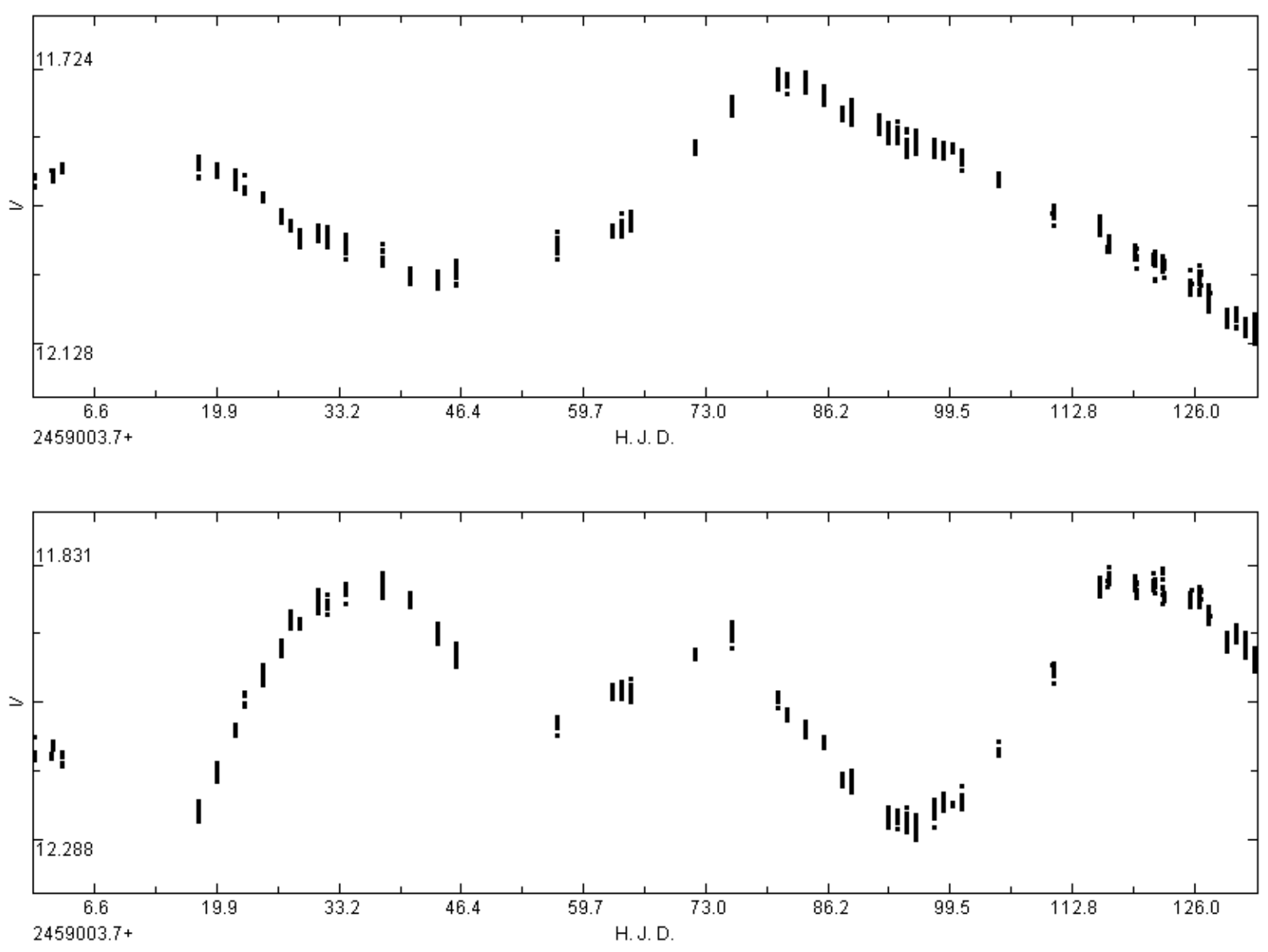

Figure 2: Light curves of the variable stars V11 (upper panel) and V17 (lower panel) obtained throughout the 2020 photometric campaign: both are semiregular red giants.

\section{Data analysis}

Once all the images had been measured and the light curves of all the variables we studied -red giants, Cepheids and RR Lyraes- obtained (Violat-Bordonau, 2021), we wanted to check if the photometric calibration procedure worked just as well using only one star, specifically L199; after re-measuring the 2020 images (741 frames), we verified that all the photometric check stars presented the same oscillations on the same dates: in Figure 3 we show the light curves of L158, L77 and L353 throughout the campaign. After the initial surprise we carefully analyzed the data files that contained the measurements of these stars. 


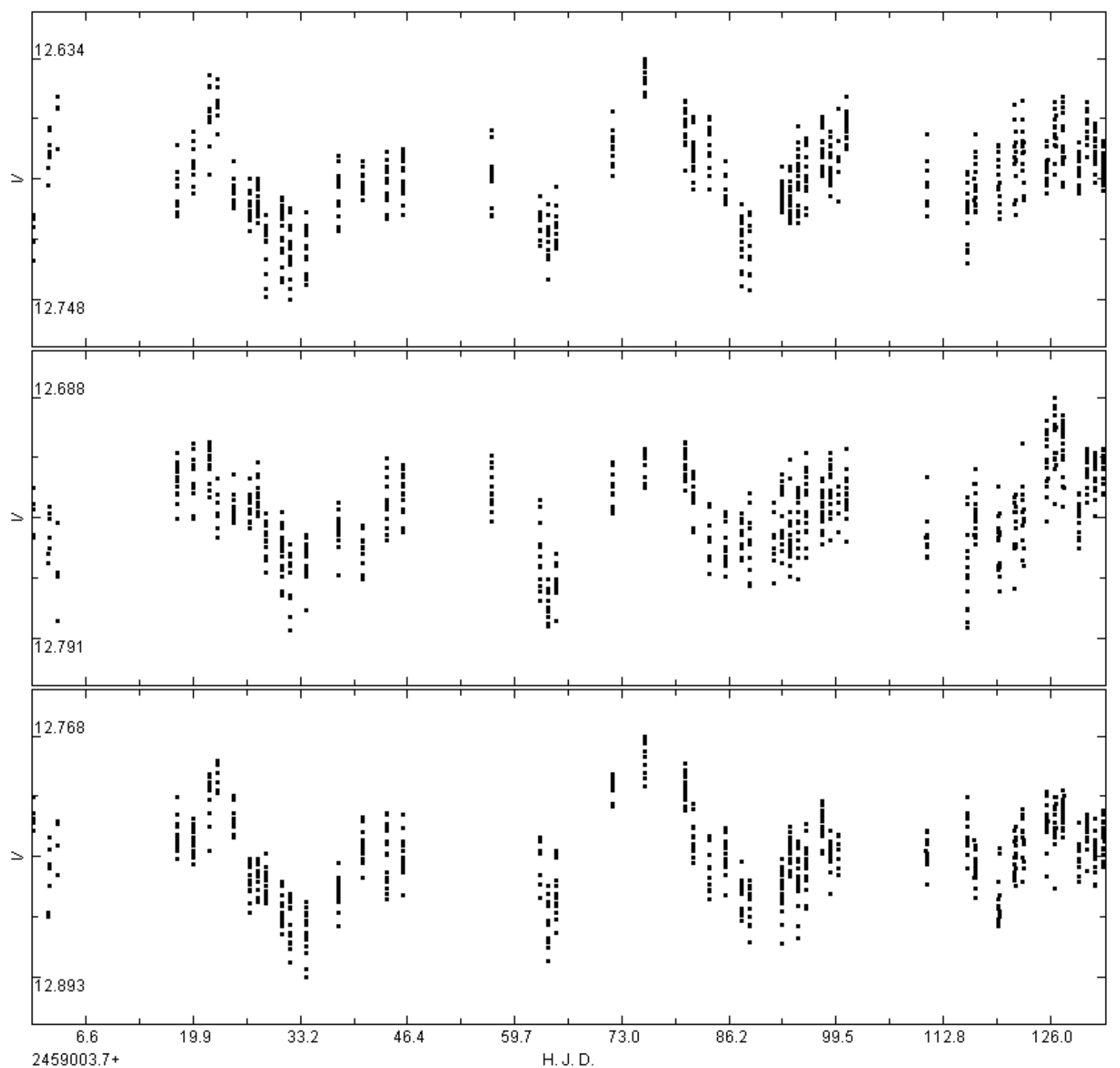

Figure 3: Light curves of the stars L158 (upper panel), L77 (central panel) and L353 (lower panel): the changes in magnitude that we see in them occur on exactly the same dates.

The periodogram analysis of the photometric data was made by $A V E^{2}$ using the Scargle algorithm (Scargle, 1982); the search in the interval 1-90 days yields a period of 27.13 days (L158, Fig. 4), 27.04 days (L77) and 27.28 days for L353: the average period is equal to 27.15 days. When we represent the light curves of these three stars with the mean period (27.15 days) we obtain quite coherent results showing very similar shapes and amplitudes (Fig. 5): we have shown the maxima in phase 0.5 to observe their shapes and amplitudes.

2 “Análisis de Variabilidad Estelar”, written by Rafael Barberá, Grupo de Estudios Astronómicos, G.E.A. 


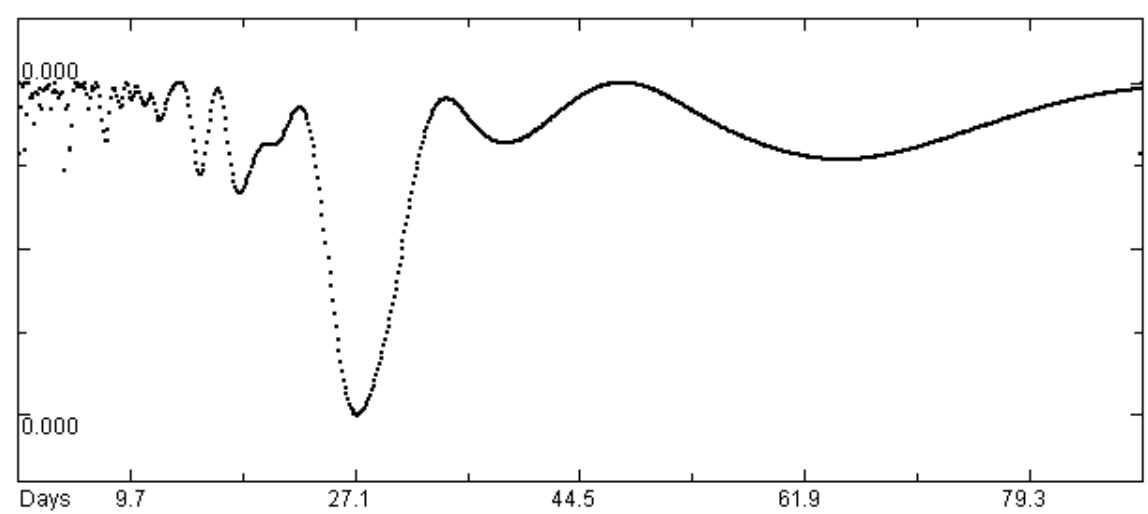

Figure 4: Scargle periodogram obtained for the interval 1-90 days of the star L158; period found: 27.13 days.

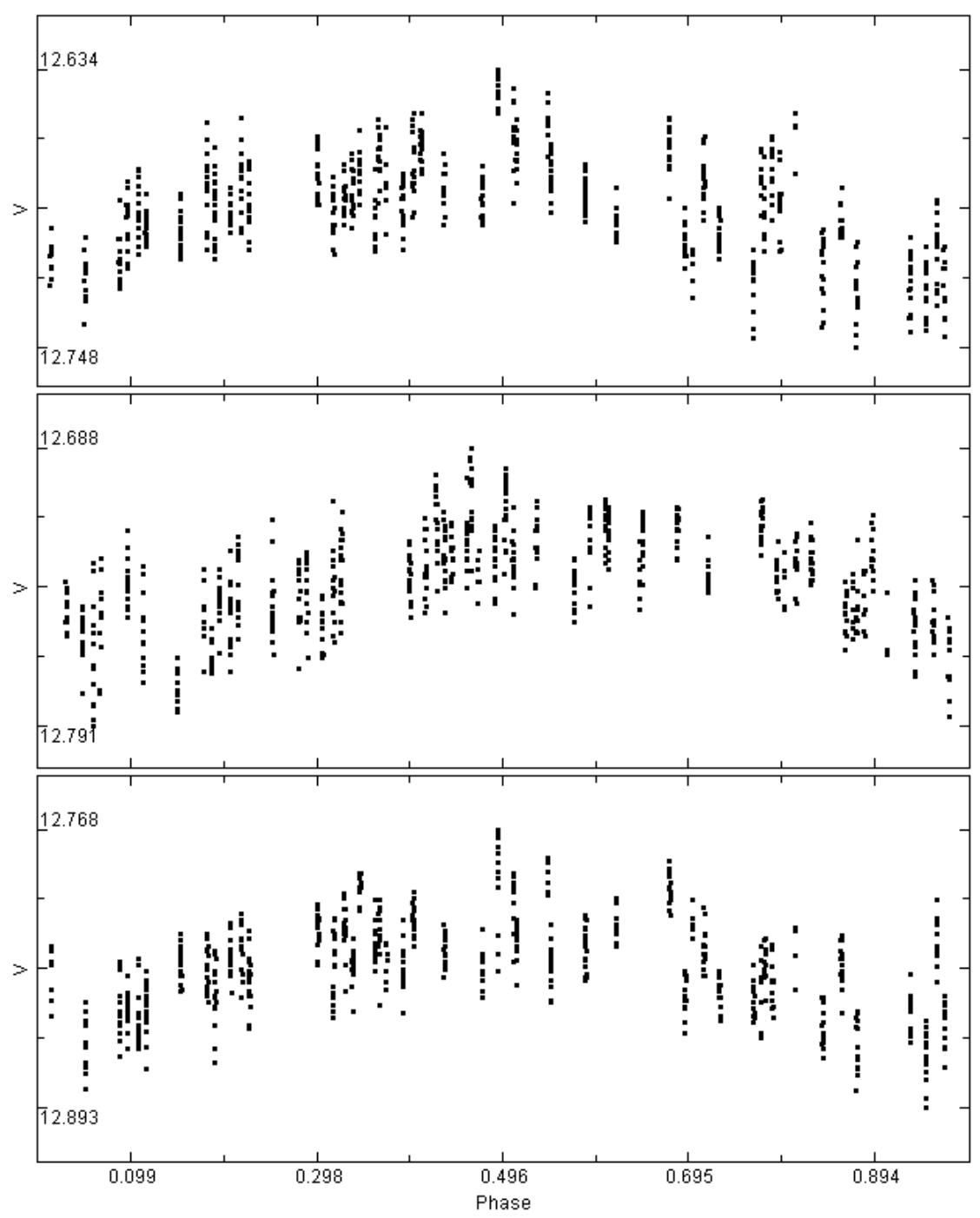

Figure 5: Light curves, in phase, of the stars L158 (upper panel), L77 (central panel) and L353 (lower panel): their shape and amplitude $(<0.130$ magnitudes in $V$ band $)$ are very similar. 
We have downloaded the photometric measurements obtained by the ZTF (Bellm \& Kulkarni, 2015) in $g$ band: we have opened and analyzed this data with FOTODIF (Fig. 6), the upper panel shows the raw measurements (between H.J.D. 2458203.901 and 2459023.741) while in the lower one, after applying the Winkler periodogram (Winkler, 1967), the period appears: $26.882 \pm 0.016$ days. When we represent the measurements with this value, we obtain the light curve in phase (Fig. 7): as in the previous cases we have drawn its maximum in phase 0.5 to see its shape and photometric amplitude. We can observe its shape, identical to the previous ones, with an amplitude that is reduced to $\sim 0.09-0.10$ magnitudes by eliminating some anomalous points: a result consistent with those we have previously presented.
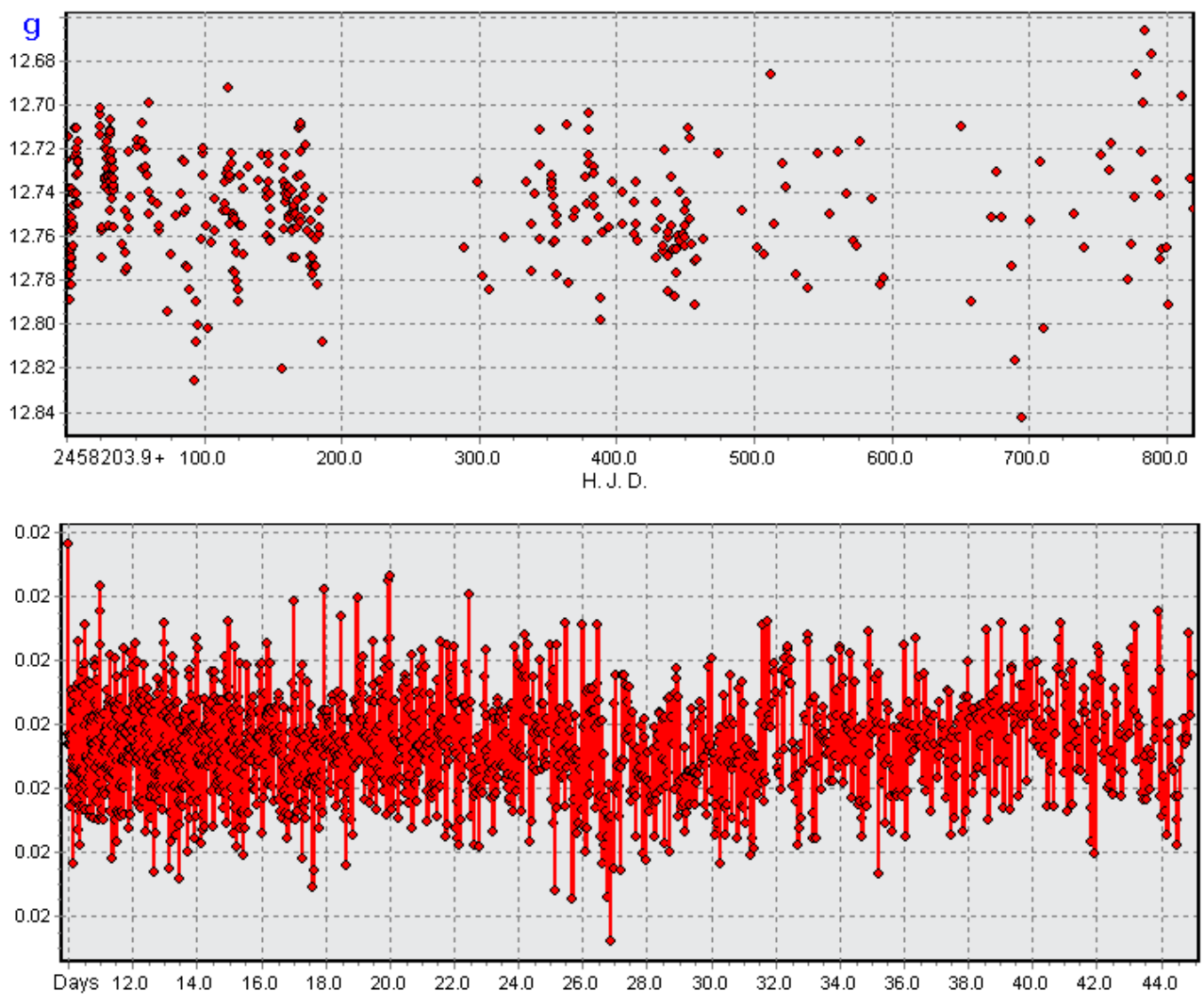

Figure 6: Photometric measurements obtained by the ZTF in $g$ band (upper panel) and most probable period in the interval 10-45 days (lower panel): the result is equal to $26.882 \pm 0.016$ days. 


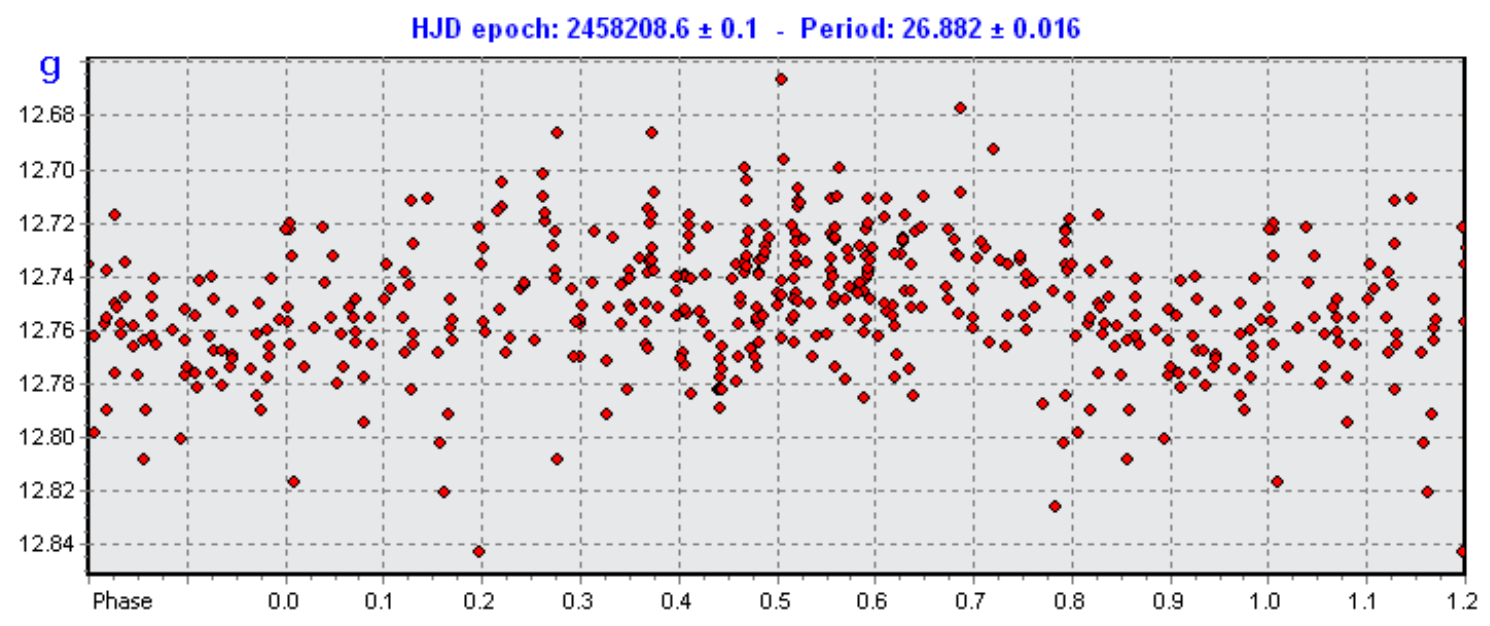

Figure 7: Light curve, in phase, plotted with the ZTF measurements using a period equal to 26.88 days: as in the previous cases, the maximum has been drawn in phase 0.5 to see its shape and amplitude.

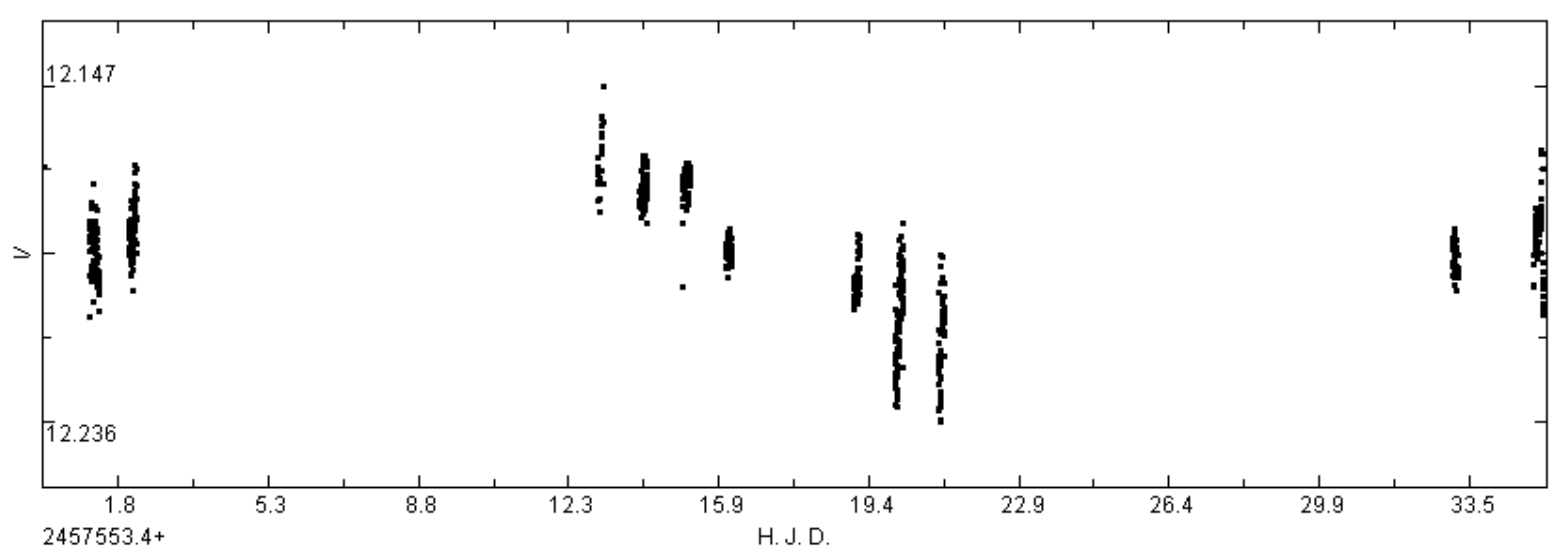

Figure 8: Photometric measurements in $V$ band provided by Dr. Deras: they show the same oscillations that we discovered in our light curves and shown in Fig. 3.

Finally, we have contacted Dr. Deras, from the Instituto de Astronomía of the Universidad Nacional Autónoma de México (who together with his collaborators discovered a large number of new variable stars in the cluster in 2019) to request his photometric measurements; once opened (Fig. 8) we verify that his data show the same oscillations that we appreciate in our light curves with an amplitude in $V$ band equal to 0.089 magnitudes. We looked for the period with $A V E$ and found three very different values: $11.03,16.34$ and 32.88 days; with none of them a good quality light curve is obtained. Using a period equal to 27 days the result is moderately consistent (Fig. 9), showing the same appearance and amplitude 


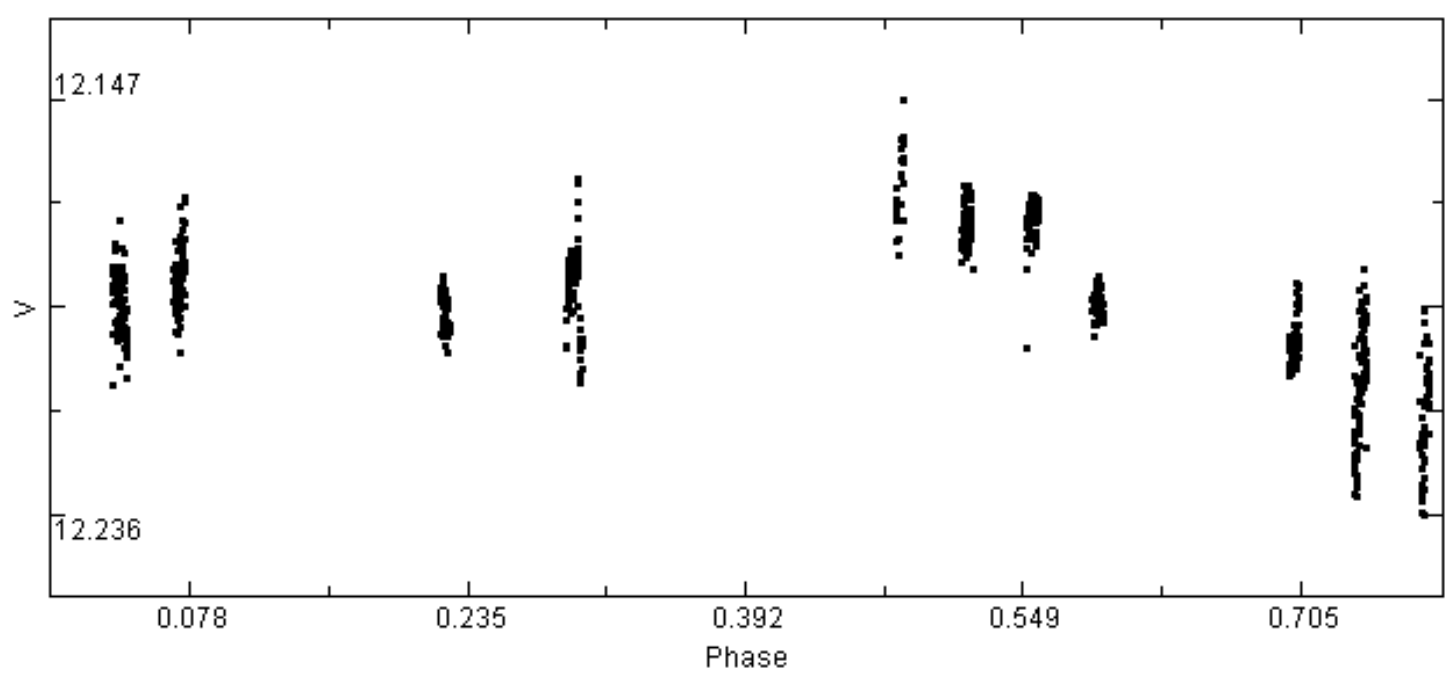

Figure 9: Light curve, in phase, plotted with the data from Deras et al. using a period equal to 27 days: it appears moderately coherent with an amplitude of 0.089 magnitudes in band $V$.

as all those previously presented.

\section{Conclusions}

From the photometric measurements of Messier 13 (NGC 6205), obtained from the Observatorio Astronómico Norba Caesarina (Cáceres, Spain) in the 2019 and 2020 campaigns, we have discovered the variability of the star L199 (TYC 2588-1386-2 and 2MASS J16413253 + 3624424), a red giant of magnitude $\sim 12.18 \mathrm{~V}$ belonging to the cluster. From our data, confirmed with photometric measurements from the ZTF and also those provided by Dr. Deras (private communication), we determined an amplitude lower than 0.08 \pm 0.03 magnitudes in Johnson $V$ band: the star changes its brightness with a period close to 27 days (probably between 25 and 30 days) but without a regular periodicity, very typical of this type of giant stars that in the case of Messier 13 oscillate between 30 and 92 days (Osborn et al., 2017).

Acknowledgements: This paper is based on observations obtained at the Observatorio Astronómico Norba Caesarina (MPC Z71) at Cáceres (Spain). We are indebted to Juan Luis González-Carballo (from the Grupo de Observadores de Supernovas) for his help in providing us with the ZTF measurements and advising us with this type of data, and Dr. Dan Deras for sharing with us his measurements and his time. We are also grateful to the referee for valuable comments. This research has also made use of the SIMBAD database, operated at CDS, Strasbourg, France, and NASA's Astrophysics Data System Abstract Service. The observational data used in this work are available upon request to Francisco Violat-Bordonau (fviolat@gmail.com). 


\section{References}

Bailer-Jones, C. A. L. et al., 2021, AJ, 161, 147: 2021AJ...161..147B

Bellm, E. C., Kulkarni, S. R., 2015, AAS Meeting \#225: 2015AAS...22532804B

Deras D., et al., 2019, MNRAS, 486, 2791: 2019MNRAS.486.2791D

Heinze, A. N. et al., 2018, AJ, 156, 241: 2018AJ....156..241H

Ludendorff, H., 1905, Publ. Astrophys. Obs. Potsdam, Vol. 15, № 50: 1905POPot..50....1L

Osborn, W., 2000, AJ, 119, 2902: 2000AJ...119.2902O

Osborn, W. et al., 2017, Acta Astronomica, 67, 131: 2017AcA...67..1310

Sandquist, E. L. et al., 2010, AJ, 139, 2374: 2010AJ....139.2374S

Scargle, J. D., 1982, ApJ, 263, 835: 1982ApJ...263..835S

Stetson, P. B. et al., 2019, MNRAS, 485, 3042: 2019MNRAS.485.3042S

Violat-Bordonau, F., 2021, web Casanchi: http://casanchi.org/ast/fotometria2019202001.htm

Winkler, L., 1967, AJ, 72, 226: 1967AJ.....72..226W 